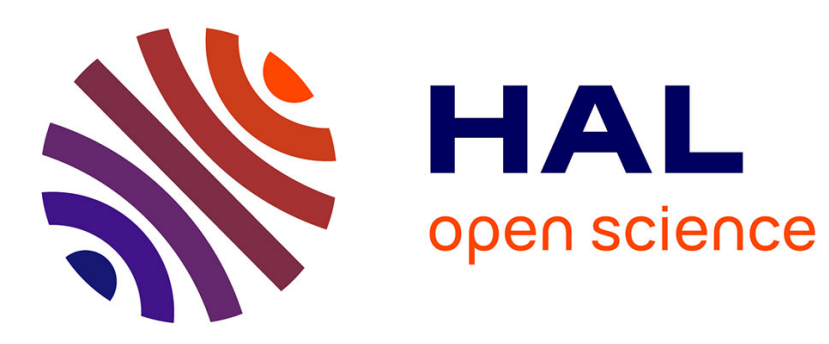

\title{
Fault diagnosis for nonlinear systems represented by heterogeneous multiple models
}

\author{
Rodolfo Orjuela, Benoît Marx, José Ragot, Didier Maquin
}

\section{To cite this version:}

Rodolfo Orjuela, Benoît Marx, José Ragot, Didier Maquin. Fault diagnosis for nonlinear systems represented by heterogeneous multiple models. Conference on Control and Fault-Tolerant Systems, SysTol'10, Oct 2010, Nice, France. pp.CDROM, 10.1109/SYSTOL.2010.5675969 . hal-00525236

\section{HAL Id: hal-00525236 \\ https://hal.science/hal-00525236}

Submitted on 11 Apr 2014

HAL is a multi-disciplinary open access archive for the deposit and dissemination of scientific research documents, whether they are published or not. The documents may come from teaching and research institutions in France or abroad, or from public or private research centers.
L'archive ouverte pluridisciplinaire HAL, est destinée au dépôt et à la diffusion de documents scientifiques de niveau recherche, publiés ou non, émanant des établissements d'enseignement et de recherche français ou étrangers, des laboratoires publics ou privés. 


\title{
Fault diagnosis for nonlinear systems represented by heterogeneous multiple models
}

\author{
Rodolfo Orjuela, Benoît Marx, José Ragot and Didier Maquin
}

\begin{abstract}
This paper proposes two observer-based FDI strategies for nonlinear systems represented by a particular class of multiple model using heterogeneous submodels. The structure of this interesting multiple model is firstly presented in order to design two kinds of state observers. The first observer, known as proportional observer (PO), is an extension of the classic Luenberger observer, in this way, it can be used to obtain an estimation of the system state. The second proposed observer, known as proportional-integral observer (PIO), makes it possible the simultaneous state and unknown input (e.g. a fault) estimation of the system under investigation. The convergence towards zero of the estimation errors provided by these observers is investigated with the help of the Lyapunov method. The $P$ observer as well as the PI observer are employed in a FDI strategy in order to accomplish the detection, the localisation and eventually the estimation of sensor faults acting on the system. These two strategies are finally validated in simulation by considering a simplified model of a bioreactor.
\end{abstract}

\section{INTRODUCTION}

Nowadays, fault detection and isolation (FDI) is increasingly integrated in many real-world applications to provide fault symptoms which can be used to take appropriate decisions when the expected behaviour of the monitored system is abnormal. Several techniques can be used to cope with the FDI problem, among them observer-based techniques are largely recognised [5], [14]. Observers are employed in a FDI framework to provide an estimation of the interesting signals to be monitored e.g. the outputs, the faults, etc. The FDI of the system is carried out by testing the time-evolution of some residual signals provided by the observer. In theory, a residual signal is null when the system behaviour is according to the expected behaviour in the normal operating conditions.

Accurate mathematical models, in the whole operating range of the system, are often necessary to accomplished the FDI. However the observer design becomes extremely arduous and even impossible when the used model is too complex. Indeed, the observer design complexity is strongly related to the choice of the model structure (linear or nonlinear) used in the modelling stage. Hence, an interesting issue is to propose state estimation techniques based on nonlinear models able to capture complex nonlinear dynamic behaviours with a simple mathematical structure to reduce

R. Orjuela is with the Laboratoire Modélisation Intelligence Processus Systèmes (MIPS) EA 2332, Université de Haute-Alsace, 12 rue des frères Lumière, F-68093 Mulhouse Cedex, France. rodolfo.orjuela@uha.fr

B. Marx, J. Ragot, D. Maquin are with the Centre de Recherche en Automatique de Nancy (CRAN), UMR 7039, Nancy-Université, 2 avenue de la forêt de Haye, F-54516 Vandœuvre, France. \{benoit.marx, jose.ragot, didier.maquin\}@ensem.inpl-nancy.fr the observer design complexity. Besides, the use of appropriated black-box identification techniques must be possible in order to obtain the parameters of such models in an experimental manner [16]. The multiple model [10] is among this interesting category of nonlinear models.

Multiple model approach [10] is an appropriate tool for modelling a large class complex nonlinear systems using a mathematical model which can be used for analysis, controller and observer design. The basis of the multiple model approach is the decomposition of the operating space of the system into a finite number of operating zones. Hence, the dynamic behaviour of the system inside each operating zone can be modelled using a simple submodel, for example a linear model. The contribution of each submodel is quantified thanks to a weighting function. Finally, the approximation of the system behaviour is performed by taking into consideration the respective contributions of the submodels via an interpolation strategy.

The interpolation of the submodels can be operated using many architectures [7], two main architectures can be distinguished among them. In the first architecture, TakagiSugeno multiple model, the set of the used submodels shares the same state space and consequently the submodels are homogeneous. In the second one, heterogeneous submodel can be used because each submodel has its own state space eventually of different dimension. Many contributions concern the analysis, the control and the state estimation of systems modelled by the first multiple model [1], [4], [10], [17]. Concerning the heterogeneous multiple model, it is already employed for dealing with the identification [11], [19] and/or control [8], [9]. However, much less studies are devoted to the state estimation and the FDI of nonlinear systems represented by this kind of model [11], [12], [18].

Heterogeneous multiple models are successfully exploited in [18] in order to design an observer-based FDI strategy (Neuro-Fuzzy Decoupling Fault Detection Scheme NFDFDS) for nonlinear systems. Interesting results are obtained in this way but the theoretical analyse of the state estimation error is not truly proposed in this work and the proof of the convergence towards zero of the estimation error is missing.

In this work, a procedure to design two kinds of observers, proportional observer ( $\mathrm{P} 0)$ and proportional-integral observer (PIO), is respectively proposed in sections III and IV. A theoretical proof of the state estimation error convergence towards zero is given using the Lyapunov method under LMI conditions [2]. Finally, in section V, two FDI strategies based on these two observers are proposed and validated in simulation by considering a simplified model of a bioreactor. 
Notations : The following notations will be used all along this paper. $P>0(P<0)$ denotes a positive (negative) definite matrix $P ; X^{T}$ denotes the transpose of matrix $X, I$ is the identity matrix of appropriate dimension and diag $\left\{A_{1}, \ldots, A_{n}\right\}$ stands for a block-diagonal matrix with the matrices $A_{i}$ on the main diagonal. Finally, we shall simply write $\mu_{i}(\xi(t))=$ $\mu_{i}(t)$.

\section{ON THE HETEROGENEOUS MULTIPLE MODEL}

The multiple model strategy is based on the basic idea that complex dynamic behaviours can be accurately represented with the help of an interpolation of simple submodels. In this paper, heterogeneous multiple model will be employed [7]. The state space representation of this multiple model is:

$$
\begin{aligned}
x_{i}(t+1) & =A_{i} x_{i}(t)+B_{i} u(t), \\
y_{i}(t) & =C_{i} x_{i}(t) \\
y(t) & =\sum_{i=1}^{L} \mu_{i}(\xi(t)) y_{i}(t),
\end{aligned}
$$

where $L$ is the number of the submodels, $x_{i} \in \mathbb{R}^{n_{i}}$ and $y_{i} \in \mathbb{R}^{p}$ are respectively the state vector and the output of the $i^{t h}$ submodel; $u \in \mathbb{R}^{m}$ is the input and $y \in \mathbb{R}^{p}$ the measured output. The matrices $A_{i} \in \mathbb{R}^{n_{i} \times n_{i}}, B_{i} \in \mathbb{R}^{n_{i} \times m}, C_{i} \in \mathbb{R}^{p \times n_{i}}$ are known and appropriately dimensioned.

The complete partition of the operating space of the system is performed using a decision variable $\xi(t)$ that is assumed to be known and real-time available (e.g. the inputs and/or exogenous signals). Notice that the contribution of each submodel is quantified by the weighting functions $\mu_{i}(\xi(t))$ that satisfy the following convex sum constraints:

$\sum_{i=1}^{L} \mu_{i}(\xi(t))=1$ and $0 \leq \mu_{i}(\xi(t)) \leq 1, \quad \forall i=1 \ldots L, \forall t$

It should be remarked that each submodel has its own state space because the blending between the submodels is performed through a weighted sum of the submodel outputs (see equation (1c)). Consequently, the dimension of the state vector $x_{i}$ of each submodel can be different and a homogeneous description of the dynamic behaviour of the system, inside each operating zone, is avoided in this way. Indeed, the submodel complexity can be well adapted according to the complexity of the system in each operating region. Hence, the number of the parameters needed to provide an accurate representation of the system under investigation can be reduced with the help of heterogeneous submodels. Therefore, this structure is well adapted for modelling strongly nonlinear systems whose structure varies with the operating regime, for example, when the complexity of the dynamic behaviour is not uniform in the operating range.

Remark 1: The outputs $y_{i}(t)$ of each submodel must be considered as intermediary modelling signals only used in order to provide a representation of the nonlinear system. Hence, they cannot be employed for driving an observer because they are not physically available and consequently no measurement is possible. Only the global output $y(t)$ of the multiple model can be used for this purpose.

\section{PROPORTIONAL OBSERVER DESIGN (PO)}

Recently, the observer design procedure of a proportional observer based on the heterogeneous multiple model has been proposed in [11], [12]. This observer takes the following architecture :

$$
\begin{aligned}
\hat{x}_{i}(t+1) & =A_{i} \hat{x}_{i}(t)+B_{i} u(t)+K_{i}(y(t)-\hat{y}(t)), \\
\hat{y}_{i}(t) & =C_{i} \hat{x}_{i}(t), \\
\hat{y}(t) & =\sum_{i=1}^{L} \mu_{i}(t) \hat{y}_{i}(t),
\end{aligned}
$$

where $\hat{x}_{i} \in \mathbb{R}^{n_{i}}$ is the estimated state vector of the $i^{t h}$ submodel, $y(t)$ the multiple model output vector, $\hat{y}(t)$ the estimated output vector provided by the observer and $K_{i} \in$ $\mathbb{R}^{n_{i} \times p}$ the $i^{t h}$ observer gain to be determined.

The use of the augmented state vector:

$$
\hat{x}(t)=\left[{\hat{x_{1}}}^{T}(t) \cdots{\hat{x_{i}}}^{T}(t) \cdots{\hat{x_{L}}}^{T}(t)\right]^{T} \in \mathbb{R}^{n}, n=\sum_{i=1}^{L} n_{i}
$$

enables to rewrite the observer equations (3) under the following compact form:

$$
\begin{aligned}
\hat{x}(t+1) & =\tilde{A} \hat{x}+\tilde{B} u(t)+\tilde{K}(y(t)-\hat{y}(t)), \\
\hat{y}(t) & =\tilde{C}(t) \hat{x}
\end{aligned}
$$

where

$$
\begin{aligned}
\tilde{A} & =\operatorname{diag}\left\{A_{1}, \cdots, A_{n}\right\} \in \mathbb{R}^{n \times n}, \\
\tilde{B} & =\left[B_{1}^{T}, \cdots, B_{n}^{T}\right]^{T} \in \mathbb{R}^{n \times m}, \\
\tilde{C}(t) & =\left[\mu_{1}(t) C_{1}, \cdots, \mu_{L}(t) C_{L}\right] \in \mathbb{R}^{p \times n}, \\
\tilde{K} & =\left[K_{1}^{T}, \cdots, K_{L}^{T}\right]^{T} \in \mathbb{R}^{n \times p} .
\end{aligned}
$$

The observer gain $\tilde{K}$ must be determined to ensure for example the exponential convergence towards zero of the state estimation error:

$$
e(t)=x(t)-\hat{x}(t)
$$

The time-evolution of the state estimation error (6) is given by:

$$
e(t+1)=A_{o b s}(t) e(t)
$$

where $A_{o b s}(t)$ is defined by

$$
A_{o b s}(t)=\tilde{A}-\tilde{K} \tilde{C}(t)
$$

The equation (7) is easily obtained by considering the timeevolution of the equation (6) and using the augmented equations of the multiple model and the observer (4).

Remark 2: The time-varying matrix $\tilde{C}(t)$ in (7) can be rewritten, using the weighting functions properties (2), as the following weighted sum of constant matrices:

$$
\tilde{C}(t)=\sum_{i=1}^{L} \mu_{i}(t) \tilde{C}_{i},
$$

where $\tilde{C}_{i}$ is a constant block matrix given by:

$$
\tilde{C}_{i}=\left[\begin{array}{lllll}
0 & \cdots & C_{i} & \cdots & 0
\end{array}\right],
$$

such that the term $C_{i}$ is found on the $i^{\text {th }}$ block column of $\tilde{C}_{i}$.

Notice that the individual design of each observer related to each submodel, using for example standard linear techniques, cannot be used to obtain the global observer (4) related to the multiple model. Indeed, the convergence of 
the state estimation error (6) is not guaranteed in this manner because the interpolation of the submodel outputs is not taken into consideration in the observer design. The interpolation of the submodel outputs for any initial conditions of the system $x(0)$ and the observer $\hat{x}(0)$ must be taken into account in the global observer design procedure. Therefore, the observer design is carried out from equation (7) using the Lyapunov method. The following theorem proposes sufficient conditions ensuring the exponential convergence of the estimation error (6).

Theorem 1: The state estimation error (6) between the multiple model (1) and the PO (3) converges exponentially towards zero if there exists a symmetric, positive definite matrix $P \in \mathbb{R}^{n \times n}$ and a matrix $G \in \mathbb{R}^{n \times p}$ solution of the constrained LMI problem:

$$
\left[\begin{array}{cc}
(1-2 \alpha) P & \tilde{A}^{T} P-\tilde{C}_{i}^{T} G^{T} \\
P \tilde{A}-G \tilde{C}_{i} & P
\end{array}\right]>0, \quad i=1, \cdots, L,
$$

for a given decay rate $0<\alpha<0.5$. The observer gain is obtained by $\tilde{K}=P^{-1} G$.

Proof. This theorem is obtained by considering a quadratic Lyapunov function:

$$
V(e(t))=e^{T}(t) P e(t), \quad P>0, \quad P=P^{T} .
$$

The exponential convergence of the estimation error is guaranteed by [2] :

$$
\exists P=P^{T}>0, \quad \alpha>0: \Delta V(e(t))+2 \alpha V(e(t))<0,
$$

where $\Delta V(e(t))=V(e(t+1))-V(e(t))$ and where $\alpha$ is the decay rate to ensure the convergence velocity. By using (12), the inequality (13) becomes:

$$
e^{T}(t+1) P e(t+1)-(1-2 \alpha) V(e(t))<0,
$$

which can be rewritten as follows considering (7):

$$
e^{T}(t)\left\{A_{o b s}^{T}(t) P A_{o b s}(t)-(1-2 \alpha) P\right\} e(t)<0,
$$

Finally, the LMIs in theorem (1) are obtained using the wellknown Schur complement [2], considering remark 2 and the weighting functions properties given by (2).

Let us notice that adequate eigenvalues placement of the observer can be obtained by an appropriate choice of the decay rate $\alpha$ in theorem 1 . For example, the asymptotic convergence of the estimation error (6) is obtained by considering $\alpha=0$.

\section{PROPORTIONAL-INTEGRAL OBSERVER DESIGN (PIO)}

In this section, unknown inputs (UI) acting on the system are considered. UI can be used, for example, to take into consideration faults acting on the system. The heterogeneous multiple model, already defined by (1), is modified as follows to take into account the unknown input $\eta(t)$ acting on the system:

$$
\begin{aligned}
x_{i}(t+1) & =A_{i} x_{i}(t)+B_{i} u(t)+D_{i} \eta(t), \\
y_{i}(t) & =C_{i} x_{i}(t) \\
y(t) & =\sum_{i=1}^{L} \mu_{i}(t) y_{i}(t)+V \eta(t),
\end{aligned}
$$

where $x_{i} \in \mathbb{R}^{n_{i}}$ and $y_{i} \in \mathbb{R}^{p}$ are respectively the state and the output of the $i^{\text {th }}$ submodel, $u \in \mathbb{R}^{m}$ is the measured input, $\eta \in \mathbb{R}^{q}$ the unknown input, $y \in \mathbb{R}^{p}$ the measured output. The matrices $A_{i} \in \mathbb{R}^{n_{i} \times n_{i}}, B_{i} \in \mathbb{R}^{n_{i} \times m}, D_{i} \in \mathbb{R}^{n_{i} \times l}, C_{i} \in \mathbb{R}^{p \times n_{i}}$ and $V \in \mathbb{R}^{p \times l}$ are known. The additional matrices $D_{i}$ and $V$ represent respectively the impact of the UI on the state and on the output e.g. a sensor or actuator fault (see section V).

The simultaneous state and UI estimation is obtained with the help of a proportional-integral observer [3], [15] given by:

$$
\begin{aligned}
\hat{x}_{i}(t+1)= & A_{i} \hat{x}_{i}(t)+B_{i} u(t)+D_{i} \hat{\eta}(t) \\
& +K_{i}(y(t)-\hat{y}(t)), \\
\hat{\eta}(t+1)= & \hat{\eta}(t)+K_{I}(y(t)-\hat{y}(t)), \\
\hat{y}_{i}(t) & =C_{i} \hat{x}_{i}(t), \\
\hat{y}(t) & =\sum_{i=1}^{L} \mu_{i}(t) \hat{y}_{i}(t)+V \hat{\eta}(t),
\end{aligned}
$$

where $\hat{x}_{i}$ is the state estimation of the $i^{t h}$ submodel, $\hat{y}(t)$ the system output estimation provided by the observer, $\hat{\eta}(t)$ the UI estimation. The observer gains $K_{i} \in \mathbb{R}^{n_{i} \times p}$ and $K_{I} \in \mathbb{R}^{q \times p}$ must be determined. This observer can be considered as a particular UI observer.

Notice that the use of the two gains $K_{i}$ and $K_{I}$ is at the origin of the "proportional-integral" terminology. Indeed, the $K_{i}$ gains ensure a proportional correction according to the output estimation error $y(t)-\hat{y}(t)$. On the other hand, the $K_{I}$ gain provides a correction in the integral loop given by the equation (17b). The UI estimation is then provided thanks to this integral action when the UI is a constant signal [3], [15].

Assumption 1: The unknown input $\eta(t)$ is assumed as constant signal: $\eta(t+1)=\eta(t)$.

The gains $K_{i}$ and $K_{I}$ of the PI observer are designed in order to ensure that the state estimation error:

$$
e(t)=x(t)-\hat{x}(t)
$$

and the unknown input estimation error

$$
\varepsilon(t)=\eta(t)-\hat{\eta}(t)
$$

converge exponentially towards zero.

The time-evolution of the state estimation error (18) is given by:

$$
e(t+1)=(\tilde{A}-\tilde{K} \tilde{C}(t)) e(t)+(\tilde{D}-\tilde{K} V) \varepsilon(t),
$$

where $\tilde{A}, \tilde{K}, \tilde{C}(t)$ are already defined in (5) and where

$$
\tilde{D}=\left[D_{1}^{T}, \cdots, D_{L}^{T}\right]^{T} \in \mathbb{R}^{n \times l} .
$$

The equation (20) is obtained by considering the both multiple model and PIO augmented equations. The time-evolution of the UI estimation error (19) is given by:

$$
\varepsilon(t+1)=\eta(t+1)-\hat{\eta}(t)-K_{I}(y(t)-\hat{y}(t))
$$

which can be simplified as follows:

$$
\varepsilon(t+1)=\varepsilon(t)-K_{I} \tilde{C}(t) e(t)-K_{I} V \varepsilon(t)
$$


according to the assumption 1 (i.e. $\eta(t+1)-\eta(t)=0$ ). Finally, equations (20) and (23) can be gathered as follows:

$$
\left[\begin{array}{l}
e(t+1) \\
\varepsilon(t+1)
\end{array}\right]=\left[\begin{array}{cc}
\tilde{A}-\tilde{K} \tilde{C}(t) & \tilde{D}-\tilde{K} V \\
-K_{I} \tilde{C}(t) & \mathrm{I}-\mathrm{K}_{\mathrm{I}} \mathrm{V}
\end{array}\right]\left[\begin{array}{l}
e(t) \\
\varepsilon(t)
\end{array}\right]
$$

which can be rewritten as

$$
e_{a}(t+1)=\left(A_{a}-K_{a} C_{a}(t)\right) e_{a}(t),
$$

where

$$
\begin{aligned}
e_{a}(t) & =\left[\begin{array}{l}
e(t) \\
\varepsilon(t)
\end{array}\right], \quad A_{a}=\left[\begin{array}{cc}
\tilde{A} & \tilde{D} \\
0 & \mathrm{I}
\end{array}\right], \quad K_{a}=\left[\begin{array}{l}
\tilde{K} \\
K_{I}
\end{array}\right], \\
C_{a}(t) & =\left[\begin{array}{ll}
\tilde{C}(t) & V
\end{array}\right] .
\end{aligned}
$$

The following theorem proposes sufficient conditions to ensure the exponential convergence of the estimation error given by the PIO (17).

Theorem 2: The state estimation error between the multiple model (16) and the PIO (17) converges exponentially towards zero if there exists a symmetric, positive definite matrix $P \in \mathbb{R}^{(n+p) \times(n+p)}$ and a matrix $G \in \mathbb{R}^{(n+p) \times p}$ solution of the constrained LMI problem:

$$
\left[\begin{array}{cc}
(1-2 \alpha) P & A_{a}^{T} P-\bar{C}_{i}^{T} G^{T} \\
P A_{a}-G \bar{C}_{i} & P
\end{array}\right]>0, \quad i=1, \cdots, L,
$$

where

$$
\bar{C}_{i}=\left[\begin{array}{ll}
\tilde{C}_{i} & V
\end{array}\right]
$$

for a decay rate given by $0<\alpha<0.5$. The observer gain is given by $K_{a}=P^{-1} G$.

Sketch of the proof. Notice the similarities between the estimation error (25) and the estimation error provided by the PO previously proposed (7). Hence, LMI conditions (27) are obtained in a similar way by considering $A_{a}, K_{a}$ and $C_{a}(t)$ instead of $A, K$ and $C(t)$.

Remark that the PIO offers some robustness degree with respect to UI varying slowly in the time i.e. $\eta_{1}(t+1) \approx$ $\eta_{1}(t)$ (see section V-D). Besides, the PIO principle can be generalised to the multi-integral case to take into account polynomial unknown inputs as recently sugested in [13].

\section{OBSERVER-BASED FDI STRATEgIES}

Two FDI strategies, based on the two P and PI observers, are proposed in the following sections. The sensor fault indicator signals provided by these FDI strategies are tested using a simplified bioreactor model.

\section{A. Bioreactor model presentation}

A bioreactor may refer to any device or system that supports a biologically active environment. The term refers to a bioreactor vessel in which runs a bio-chemical reaction. A biological reaction that normally involves three kinds of variables: biomass (e.g. micro-organisms), substrate (e.g. carbon sources in the diet) and the biomass production (e.g. enzymes).

The dynamic behaviour of a continuous bioreactor homogeneously mixed (completely mixed) and limited by a single substrate, reaction of type $S(t) \rightarrow X(t)$, can be described using the following nonlinear model [6]:

$$
\begin{aligned}
\dot{S}(t) & =D(t)\left(S_{i n}(t)-S(t)\right)-k r(t), \\
\dot{X}(t) & =-D(t) X(t)+r(t),
\end{aligned}
$$

where $S(t)$ and $X(t)$ are respectively the concentration of the carbon substrate rate $S(t)$ and the biomass rate $X(t)$, where $D(t)>0$ is the dilution rate, $k$ a coefficient of productivity, $S_{i n}(t)$ is the rate of substrate feed concentration and $r(t)$ the reaction velocity (i.e. the biomass production). The reaction velocity rate $r(t)$ can be characterised by the expression:

$$
r(t)=\mu_{\max } S(t) X(t) /\left(K_{s}+S(t)\right),
$$

where $\mu_{\max }$ and $K_{s}$ are two constant which represent respectively the maximum specific growth rate and a saturation constant. The parameters used in the simulation are $\mu_{\max }=$ $0.33 \mathrm{~h}^{-1}, K_{s}=5 \mathrm{gl}^{-1}, k=20$. The rate of substrate feed concentration is considered constant $S_{i n}=20 \mathrm{gl}^{-1}$ and the dilution rate inside the range of variation $D \in\left[\begin{array}{ll}0.0 & 0.22\end{array}\right] \mathrm{h}^{-1}$. It can be noted that the considered bioreactor presents a nonlinear dynamic behaviour in this operating range.

\section{B. Multiple model representation of the bioreactor}

The goal of this section is to represent the dynamic behaviour of the bioreactor (29) with the help of a heterogeneous multiple model (1) in an experimental manner. Two sequences of pseudo-measures of the carbon substrate rate $S(t)$ and the biomass rate $X(t)$ are available to accomplished the identification and the validation tasks. These measures are generated by considering the dilution rate as a piecewise input signal with random amplitude and duration.

The decision variable $\xi(t)$ is here the input signal $u(t)$. This arbitrary choice is easily justified on the basis that the input signal drives the system through the different operating points i.e. the operating modes. The operating space of the bioreactor is decomposed into two operating regions according to the static characteristic of the bioreactor outputs. The associated weighting function of each operating region is obtained from the normalisation of Gaussian functions:

$$
\begin{aligned}
& \mu_{i}(\xi(t))=\omega_{i}(\xi(t)) / \sum_{j=1}^{L} \omega_{j}(\xi(t)), \\
& \omega_{i}(\xi(t))=\exp \left(-\left(\xi(t)-c_{i}\right)^{2} / \sigma^{2}\right),
\end{aligned}
$$

where $c_{1}=0.02, c_{2}=0.20$ and $\sigma=0.247$. The parameters of the submodels are identified according to the identification procedure proposed in [11].

As can be see from figure 1 , the identified multiple model (namely M.M.) provides a good representation of the global nonlinear behaviour of the bioreactor (namely Bio). It should however be noted that a small discrepancy between the dynamic behaviour of the bioreactor and the multiple model appears in the transitional phases. Indeed, the multiple model fails to fully follow the transient behaviour of the system. However, this representation with a reduced number of sub-models (it only has two submodels) can be used with success for the FDI purpose as shown in the following sections. Remark that the multiple model representation of 
the bioreactor, obtained in this section, is only used in order to design appropriated PO and PIO. These observers are however driven by the inputs/outputs of the bioreactor (29).
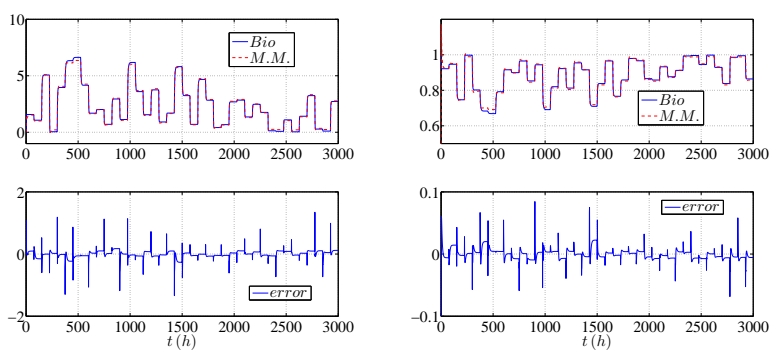

Fig. 1. Time-evolution of the carbon substrate rate $S(t)$ (left) and biomass rate $X(t)$ (right)

\section{Residual signal generation using $P$ observers}

The residual fault generation based on observers is accomplished through the estimation of system outputs using measurable signals and the model of the system. The FDI procedure is performed by analysing the time-evolution of the residual signals obtained by the comparison between the measured outputs and the estimated outputs [5], [14]. In theory, the residual signals (i.e. the estimation error) is null under normal operating conditions of the system. The residual signal structuring, in order to generated appropriated fault indicators, can be obtained by replacing the use of only one observer by the use of a bank of observers where each observer is driven by a partial set of the available signals. The well known Dedicated Observer Scheme (DOS) [5], [14] can be employed in order to obtain structured residual signals for sensor faults isolation and detection. In this case, the $i^{\text {th }}$ observer is driven by all inputs and the $i^{\text {th }}$ output of the system.

Here, only sensor faults acting on the bioreactor outputs, $S(t)=y_{1}(t)$ and $X(t)=y_{2}(t)$, are considered. They are respectively noted $\eta_{1}(t)$ and $\eta_{2}(t)$. The DOS strategy related to the sensor fault problem of the bioreactor is accomplished with the help of three PO: the first observer $\left(\mathrm{PO}_{1}\right)$ is driven by the input and the first output $y_{1}$, the second observer $\left(\mathrm{PO}_{2}\right)$ by the input and the second output $y_{2}(t)$ and the third observer $\left(\mathrm{PO}_{3}\right)$ by the input and the two outputs $y=$ $\left[\begin{array}{ll}y_{1} & y_{2}\end{array}\right]^{T}$. In the sequel, $r_{i, j}$ is the fault indicator signals obtained from the error between the $i^{\text {th }}$ output of the system and the $i^{t h}$ output estimated by the $j^{\text {th }}$ observer. The isolation of a sensor fault $\eta_{i}$ is performed via an incidence matrix which takes into account the time-evolutions of the residual signals according to the sensor faults acting on the system (see table I). In this matrix, a " 1 " element indicates that the residual signal $r_{i, j}$ is sensitive to the fault $\delta_{i}$ while a " 0 " element indicates that the residual signal $r_{i, j}$ does not respond to the fault $\delta_{i}$. Finally, the symbol "?" indicates that no decision can be taken only based on this residual.

The incidence matrix is built according to the following discussion [12]:

1) The output $y_{1}$ is corrupted by a sensor fault $\eta_{1} \neq 0$ but the output $y_{2}$ is free of fault $\eta_{2}=0$. The state estimation performed by the $\mathrm{PO}_{1}$ is then corrupted and the direction of the residual signals $r_{i, 1}$ are unknown due to the non linearity of the observer and since compensation phenomena which can appear. Hence, the value of residual signals $r_{i, 1}$ may remains null in presence of faults. Therefore, "?" element indicates that no decision can be taken.

2) On the other hand, the state estimation performed by the $\mathrm{PO}_{2}$ is correctly performed because this observer is driven by the output $y_{2}$ free of fault. Therefore, the residual signal $r_{1,2}$ is undoubtedly sensitive to a fault $\eta_{1}$ (" 1 " element is used) whereas $r_{2,2}$ is not sensitive to this same fault (" 0 " element is used). Hence, this configuration will be exploited to conclude about the presence of a fault on the output $y_{1}$.

3) The $\mathrm{PO}_{3}$ is simultaneously driven by the two outputs and consequently the state estimation is corrupted ("?" element is used).

The second line of the incidence matrix can be built in a similar way.

\begin{tabular}{c||c|c|c|c|c|c|}
\cline { 2 - 7 } \multicolumn{1}{c|}{} & \multicolumn{2}{c|}{$\mathrm{PO}_{1}$} & \multicolumn{2}{c|}{$\mathrm{PO}_{2}$} & \multicolumn{2}{c|}{$\mathrm{PO}_{3}$} \\
\cline { 2 - 7 } \multicolumn{1}{c|}{} & $r_{1,1}$ & $r_{2,1}$ & $r_{1,2}$ & $r_{2,2}$ & $r_{1,3}$ & $r_{2,3}$ \\
\hline \hline$\eta_{1}$ & $?$ & $?$ & 1 & 0 & $?$ & $?$ \\
\hline$\eta_{2}$ & 0 & 1 & $?$ & $?$ & $?$ & $?$ \\
\hline
\end{tabular}

INCIDENCE MATRIX

The considered faults acting on the system outputs of the bioreactor (29) are step signals of amplitude equal to $10 \%$ of the maximal amplitude of each output. The sensor fault $\eta_{1}$ appears on the output $y_{1}(t)$ at $t=1880$ and vanishes at $t=2380$. The sensor fault $\eta_{2}$, acting on the output $y_{2}(t)$, appears at $t=625$ and vanishes at $t=1240$. The FDI tasks are accomplished by monitoring the residual signals $r_{i, 1}$ and $r_{i, 2}$ according to the incidence matrix $\mathrm{I}$.

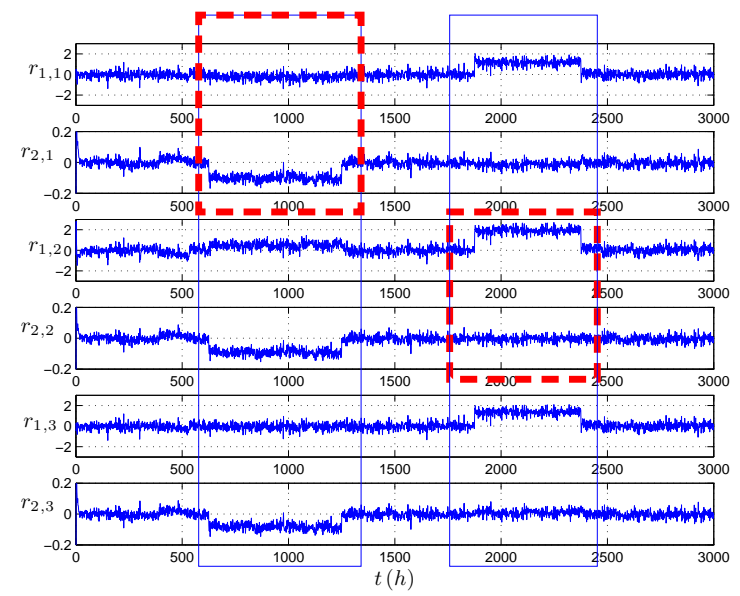

Fig. 2. Time-evolution of residual signals $r_{i, j}$

The figure 2 shows the time-evolution of the residual signals obtained from the DOS strategy. During the absence of faults $(t<625$ or $t>2500)$ the residual signals are statistically null. In the time-interval $625 \leq t \leq 1240$, the residual signals $r_{1,1}$ and $r_{2,1}$ are according to the fault signature $\eta_{2}$ 
on the output $y_{2}$. This information is also verified by the residual signals generated by the two observers. During the time-interval $1880 \leq t \leq 2380$, the residual signals $r_{1,2}$ and $r_{2,2}$ are according to the fault signature $\eta_{1}$ on the output $y_{1}$. Notice however that the isolation of sensor faults acting simultaneously on the outputs becomes impossible. In order to avoid this problem, the PIO proposed in section IV can be used for residual signal generation.

\section{Residual signal generation using PI observers}

Unknown input observers are employed as an alternative of PO to generate structured fault signals. Indeed, the PIO previously proposed is a particular class of unknown input observer which makes it possible the simultaneously state and output estimations. Hence, the unknown input estimation provided by this observer can be directly used as a residual signal, i.e. sensor fault indicator, because the sensor faults are considered as unknown inputs to be estimated.

Here, the matrices $\tilde{D}$ and $V$ used to take into account the impact of the faults on the states and on the outputs respectively are given: $\tilde{D}=0_{(4 \times 2)}$ and $V=\operatorname{diag}\{1,1\}$ because only sensor faults are considered.
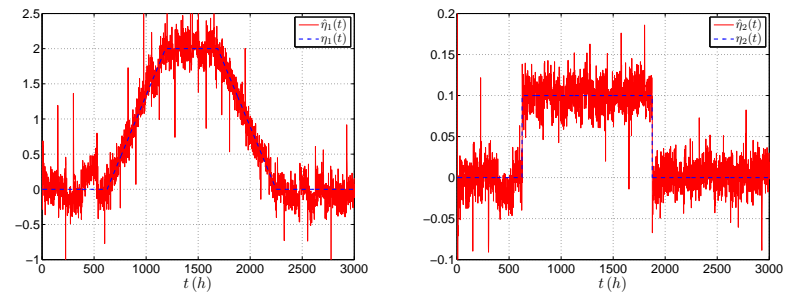

Fig. 3. Comparison between sensor faults $\eta_{i}$ and their estimated $\hat{\eta}_{i}$

The figure 3 shows the injected sensor faults $\eta_{i}$ (dashed line) acting on the bioreactor outputs and their estimates $\hat{\eta}_{i}$ (plain line). Remark that the time-evolution of the estimated faults $\hat{\eta}_{i}$ are according to the time-evolution of the sensor faults. Hence, the sensor fault isolation can be well accomplished even if simultaneous sensor faults appear in the outputs at $t=620$. The sensor faults estimated in this manner can then be considered in a FDI as fault indicators (i.e. residual signals). The FDI task (detection, isolation and identification) can then be carried out according to the timeevolution of the unknown input estimation provided by the PIO. Let us notice that the proposed PIO is able to provided fault estimation of time-varying faults. Indeed, the considered fault $\eta_{1}(t)$ is not truly constant but varying slowly in the time.

\section{CONCLUSION}

This paper shows how observers for nonlinear systems represented by heterogeneous multiple models can be designed and employed in a FDI strategy. The state estimation problem is tacked with the help of two kinds of observers: proportional and proportional-integral observers The PO provides the state estimation of the system under investigation. The PIO makes it possible the simultaneous state and unknown input estimations, in this way this observer is an unknown input observer. Sufficient conditions, under LMI form, are established to ensure the exponential convergence of the estimation errors. We have shown how the two proposed observers can be exploited in a FDI framework of nonlinear systems. Two FDI strategies are proposed for detection, isolation and identification. The first strategy uses the wellknown principle of a bank of observers where each observer is devoted to a particular fault. The second one takes directly into account the fault estimation provided by the proposed unknown input PIO. They are validated through a simulation example of a bioreactor.

\section{REFERENCES}

[1] R. Babuska. Fuzzy Modeling for Control. Kluwer Academic Publishers, 1998.

[2] S. Boyd, L. El Ghaoui, E. Feron, and V. Balakrishnan. Linear Matrix Inequalities in System and Control Theory. SIAM Studies in Applied Mathematics 15, 1994.

[3] K.K. Busawon and P. Kabore. Disturbance attenuation using proportional integral observers. International Journal of Control, 74(74): 618-627, 2001.

[4] M. Chadli,A. Akhenak, J. Ragot, D. Maquin. On the design of observer for unknown inputs fuzzy models. International Journal of Automation and Control, 2(1): 113-125, 2008.

[5] S.X. Ding. Model-based Fault Diagnosis Techniques. Springer-Verlag, 2008.

[6] I. Dunn, E. Heinzle, J. Ingham, and J. Penosil. Biological Reaction Engineering: Dynamic Modelling Fundamentals with Simulation Examples. Wiley-VCH Verlag GmbH \& Co. KGaA, 2005.

[7] D. Filev. Fuzzy modeling of complex systems. International Journal of Approximate Reasoning, 5(3): 281-290, 1991.

[8] S. García-Nieto, M. Martínez, X. Blasco, and J. Sanchis. Nonlinear predictive control based on local model networks for air management in diesel engines. Control Engineering Practice, 16(12): 1399-1413, 2008.

[9] G. Gregorcic and G. Lightbody. Control of highly nonlinear processes using self-tuning control and multiple/local model approaches. In 2000 IEEE International Conference on Intelligent Engineering Systems, INES 2000, Portoroz, Slovenia, 2000.

[10] R. Murray-Smith and T.A. Johansen. Multiple model Approaches to Modelling and Control. Taylor \& Francis, 1997.

[11] R. Orjuela. Contribution à l'estimation d'état et au diagnostic des systèmes représentés par des multimodèles. $\mathrm{PhD}$. Thesis, Institut National Polytechnique de Lorraine, France, 2008.

[12] R. Orjuela, B. Marx, J. Ragot, and D. Maquin. State estimation for nonlinear systems using a decoupled multiple mode. International Journal of Modelling Identification and Control, 4(1): 59-67, 2008.

[13] R. Orjuela, B. Marx, J. Ragot, and D. Maquin. On the simultaneous state and unknown input estimation of complex systems via a multiple model strategy. IET Control Theory and Applications, 3(7): 877-890, 2009.

[14] R. J. Patton, P. Frank, and N. Clark. Issues of Fault Diagnosis for Dynamic systems. Springer-Verlag, 2000.

[15] D. Söffker, T. Yu, and P. Müller. State estimation of dynamical systems with nonlinearities by using proportional-integral observer. International Journal of Systems Science, 26(9): 1571-1582, 1995.

[16] J. Sjöberg, Q. Zhang, L. Ljung, A. Benveniste, B. Delyon, P. Glorennec, H. Hjalmarsson, and A. Juditsky. Nonlinear black-box modeling in system identification: a unified overview. Automatica, 31(12): 1691$1724,1995$.

[17] K. Tanaka and H. Wang. Fuzzy Control Systems Design and Analysis. John Wiley \& Sons, INC., 2001.

[18] F. J. Uppal, R. J. Patton, and M. Witczak. A neuro-fuzzy multiplemodel observer approach to robust fault diagnosis based on the DAMADICS benchmark problem. Control Engineering Practice, 14(6): 699-717, 2006.

[19] A. N. Venkat, P. Vijaysai, and R. D. Gudi. Identification of complex nonlinear processes based on fuzzy decomposition of the steady state space. Journal of Process Control, 13(6): 473-488, 2003. 